\title{
SWALLOWING DISORDERS IN CLINICAL PRACTICE: FUNCTIONAL ANATOMY, ASSESSMENT AND REHABILITATION STRATEGIES
}

\author{
Stanescu Ioana ${ }^{1 / 3}$, Dogaru Gabriela ${ }^{2 / 3}$ \\ 'University of Medecine and Pharmacy "Iuliu Hatieganu” Cluj Napoca, Department of Neurosciences \\ 2 University of Medecine and Pharmacy "Iuliu Hatieganu" Cluj Napoca, Department of Physical \\ Medecine and Rehabilitation \\ ${ }^{3}$ Clinical Rehabilitation Hospital Cluj Napoca
}

\begin{abstract}
Swallowing is a complex process consisting in transporting food from mouth to the stomach; it involves voluntary and reflex activity of more than 30 nerves and muscles, requiring complex neuromuscular coordination and brainstem and cortical centers for controle.

Dysphagia is defined as a alteration in the swallowing process, which cause difficulty in transporting saliva and aliments from the mouth trough the pharynx and esophagus into the stomach . It is a frequent symptom, affecting especially old people, people with neurological diseases, cancers of head and neck or severe reflux . Dysphagia can result from a wide variety of functional or structural deficits of the oral cavity, pharynx, larynx or esophagus, which could e caused by neurological conditions. Dysphagia carries serious health risks: malnutrition, dehydration, increase risk of infections. Effective dysphagia management requires an interdisciplinary approach; the goal of rehabilitation is to identify and treat abnormalities of swallowing while maintaining safe and efficient nutrition.
\end{abstract}

KEY WORDS: swallowing, dysphagia, functional anatomy, rehabilitation, neuroplasticity

Swallowing is a complex process consisting in transporting food from mouth to the stomach. Eating and swallowing are complex behaviors involving volitional and reflexive activities of more than 30 nerves and muscles, requiring complex neuromuscular coordination and brainstem and cortical centers for controle (1). They have two crucial biological features: food passage from the oral cavity to stomach and airway protection.

Dysphagia is defined as a alteration in the swallowing process, which cause difficulty in transporting saliva and liquid or solid aliments from the mouth trough the pharynx and esophagus into the stomach (2). It is a frequent symptom, affecting around $15 \%$ of hospital inpatients, especially old people, people with neurological disease, with cancers of head and neck and people with severe reflux (3). Dysphagia can result from a wide variety of functional or structural deficits of the oral cavity, pharynx, larynx or esophagus. Predisposing factors for difficulty in swallowing could be anatomical, psychological, muscular, and many neurological diseases (4).

Clinical importance of dysphagia: This symptom affects a person's ability to remain well nourished and hydrated and increases the risks of illnesses. Dysphagia carries serious health risks: malnutrition, dehydration, lengthening of healing, increase risk of infections, impairment in mental and physical condition (2). Effective dysphagia management requires an interdisciplinary approach and can make a huge difference to the quality of life experienced by the person with dysphagia. The goal of dysphagia rehabilitation is to identify and treat 
abnormalities of feeding and swallowing while maintaining safe and efficient alimentation and hydration.(5)

Symptoms and signs of swallowing disorders: The first symptom of dysphagia is the appearance of a cough reflex during or after ingestion of food or liquids. In slight disorders, swallowing function is sufficiently compensated, and the patients has few or no symptoms, being able to compensate and obtain a safe deglutition. In severe situations, suffocation or severe cough accesses appear during meals. The swallowing problems could be revealed only by pulmonary complications, as aspiration pneumonia (post-prandial remitting type fever), severe condition especially for an immunocompromised patient (2). In patients with acquired brain damages $40 \%$ of deaths are caused by septic complications of swallowing disturbances. (6).
Assessment of swallowing disorders includes clinical and paraclinical methods.

Clinical evaluation should begin with assessment of patient's eating habits, monitoring the amount of food reflux in the mouth after swallowing, testing of pharyngeal and cough reflexes, ability to produce a voluntary cough, ability to controle posture and movements of the head, and a complete neurological and ORL examination. Referral to a gastroenterologist is warranted if the patient has likely esophageal dysphagia.

Scales have been developed for the clinical assessment of dysphagia severity. The Penetration Aspiration Scale is an ordinal scale that evaluates depth, response, and clearance of airway invasion to determine severity (table 1); was designed by Rosenbek for patients with dysphagia (13).

Table 1. Penetration-Aspiration Scale.

\begin{tabular}{|l|l|l|l|}
\hline Aspiration Risk & Score & Classification & Description \\
\hline No risk & 1 & Normal & No airway invasion. \\
\hline No risk & 2 & Mild & Bolus enters into airway with clearing. \\
\hline Risk of aspiration & 3 & Moderate & $\begin{array}{l}\text { Bolus enters into airway without } \\
\text { clearing }\end{array}$ \\
\hline & 4 & Moderate & $\begin{array}{l}\text { Bolus contacts vocal cords with airway } \\
\text { clearing. }\end{array}$ \\
\hline $\begin{array}{l}\text { Positive } \\
\text { aspiration }\end{array}$ & 5 & Moderate & $\begin{array}{l}\text { Bolus contacts vocal cords without } \\
\text { airway clearing. }\end{array}$ \\
\hline & 7 & Severe & $\begin{array}{l}\text { Bolus enters trachea and is cleared into } \\
\text { larynx or out of airway. }\end{array}$ \\
\hline & 8 & Severe & $\begin{array}{l}\text { Bolus enters trachea and is not cleared } \\
\text { despite attempts. }\end{array}$ \\
\hline
\end{tabular}


Paraclinical investigation consists in barium esophagography, videofluoroscopy, flexible endoscopic examination (FEES), ultrasound examination, manometry, electromyography, 24 hour $\mathrm{pH}$ monitoring, scintigraphy. Through the FEES, physicians can identify the phase of swallowing process which is damaged.

Assessment of swallowing disorders should be done by a multidisciplinary team, which includes ear-nose-throat (ENT) specialist, speech therapist, neurologist, dentist, or gastroenterologist.

Classification of swallowing disorders: Swallowing disorders can be classified into oropharingeal (superior) dysphagia and esophageal (inferior) dysphagia (7) (8). Most oropharyngeal dysphagia is of neurologic origin, while esophageal dysphagia is caused by gastroenterologic pathology.

Neurologic dysphagia may be caused by a disease of muscles or neuromuscular junction, or by lesions of peripheral nerves talking part in swallowing process, or lesions at the upper level of motor and sensory brainstem nuclei involved in swallowing or even at supranuclear level.

The background for neurorehabilitation strategies recently developed for swallowing disorders is the functional anatomy of swallowing process, which will further be discussed with its anatomical and physiological considerations.

a). Anatomic considerations: To understand rehabilitation strategies for dysphagic patients, the knowledge of neurologic structures and pathways that govern swallowing is crucial. In the 1980 s swallowing was thought to be "automatic" and reflex, mediated at brainstem level. Current research has changed this concept, swallowing is considered a "patterned response", mediated by complex neurophysiological processes.

Initiation of swallowing needs activation of thermo, touch, pressure or/ and chemo- receptors and sensory fibers of the oropharynx, which send taste and sensory information to five pairs of cranial nerves
(V, VII, IX, and fibers shared by X and XI). This information is then transferred to groups of nuclei in the brainstem: nucleus tractus solitarius (NTS), nucleus ambiguus (NA) and the area located in the surrounding reticular formation. Recent neuroimaging findings support that these nuclei need supramedullary input to enable initiation of motor commands, which are sent through six pairs of cranial nerves (V, VII, IX, fibers shared by $\mathrm{X}$ and XI, and XII) to the end organs, i.e. the oropharyngeal muscles.

The main muscles involved in swallowing are masticatory muscles, mylohyoid, tensor veli palatini, digastrics, stylohyoid, stylopharyngeus, levator veli palatine, palatopharyngeous, salpingopharyngeous, intrinsic laryngeal muscles, cricopharyngeus, pharyngeal constrictors, intrinsic tongue muscles, hyoglossus, geniohyoid, genioglossus, styloglossus, tyrohyoid.

Supranuclear control of swallowing process have been studied by functional MRI (14). Pharyngeal components of swallowing are controlled mainly by subcortical networks, while oral phases, which are voluntary, are under cortical controle. During voluntary swallowing, fMRI shows activation in bilateral neural networks, including primary sensorimotor cortex, supplementary motor area, prefrontal cortex, cyngulate gyrus, insula, superior temporal gyrus (14).

b). Physiological considerations : Swallowing is a very complex physiologic mechanism, which implies voluntary contractions of the oropharingeal muscles and involuntary contractions of visceral esophageal muscles. It is a cyclical function, that recurs about 2000 times for day with an average duration of $1 \mathrm{sec}$ (2). The swallowing process was commonly divided into oral, pharyngeal, and esophageal stages according to the location of the bolus. The oral stage was later subdivided into oral preparatory and oral propulsive stages, and the four stage model was established (9), (10). The movement of the food in the oral cavity and to the oropharynx differs between 
solid food and liquid.

1. The oral preparatory phase is totally voluntary. The liquid bolus is held in the anterior part of the floor of the mouth or on the tongue surface; for solid food the tongue carries the bolus to the post-canine region and rotates laterally, placing the food onto the occlusal surface of lower teeth for food processing; food particles are reduced in size by mastication and softened by salivation until the food consistency is optimal for swallowing. Chewing consists in cyclic movements of the tongue and jaw coordinated with movements of cheek, soft palate and hyoid bone (9).

2. The oral propulsive phase involves transportation of the bolus by oral and tongue muscles contractions towards the soft palate and then to the pharynx. For liquids the tongue tip rises, the tongue moves upward, squeezing the liquid bolus back along the palate and into the pharynx. For solid food, the anterior tongue surface first contacts the hard palate, squeezing the triturated food back along the palate to the oropharynx.

3.The pharyngeal phase is involuntary; the muscle elevator of the soft palate contracts and contacts the lateral and posterior walls of the pharynx, closing the nasopharynx; the pharyngeal constrictor muscles contract, squeezing the bolus downward. The vocal folds close to seal the glottis, the epiglottis tilts backward to seal the laryngeal vestibule. Safe bolus passage in the pharynx without aspirating food is critical in human swallowing, so the former mechanisms are destinated to airway protection. Opening of the upper esophageal sphincter (inferior pharyngeal constrictor muscles, cricopharyngeous muscle and most proximal part of the esophagus) finally causes bolus entry into the esophagus. (5)

4. The esophageal phase consists by peristaltic waves advancing in the direction of the stomach, where the lower esophagian sphincter relaxes and permits food entering into the stomach. This stage is under controle of autonomic nervous system. (2).
Eating, swallowing and breathing are tightly coordinated. Swallowing is dominant to respiration in normal individuals. Breathing ceases briefly during swallowing, not only because of the physical closure of the airway by elevation of the soft palate and tilting of the epiglottis, but also of neural suppression of respiration in the brainstem (11).

Causes of swallowing disorders: Palmer (12) divided the etiology in structural abnormalities of the oropharynx /esophagus (Zenker's diverticulum, congenital malformations as cleft or palate lip, cervical osteophytes, esophagian strictures, teeth loss, xerostomia, head and neck cancers), neurological diseases, functional disorders (psychogenic dysphagia) and iatrogenic causes (chemoradiation therapy for headand-neck cancer).

Dysphagia is a common problem encountered in many neurological diseases, such as strokes (brainstem, cerebellar strokes, hemispheric strokes - ischemic or hemorrhagic-), multiple sclerosis, neurodegenerative diseases (Parkinson disease, dementias, motor neuron diseases), brain tumors, poliomyelitis, polyradiculoneuropathies, myasthenia gravis, myopathies (7), (12). Studies have shown that more than a half of patients with stroke will develop swallowing disorders in the acute phase (9), and also, patients with traumatic brain injuries present swallowing disorders which are responsible for septic complications. Swallowing impairments carry serious health consequences if they are not recognized or inappropriately managed.

Treatment of swallowing disorders depends on the cause which was identified. The goal is to make patient able for a safe oral feeding (1) by preventing aspiration, to restore the lost reflex function and mental scheme and to retrieve the nutritional status (2). Neurologic dysphagia treatment is difficult, long lasting and often unsatisfactory. First it should include treatment of the neurologic cause, if possible. 
The neurologist should set the reabilitative treatment, with the aid of the speech therapist and of the ENT specialist. The choice of the rehabilitation program should be individualized, based on the results of clinical and paraclinical assessments.

There are two rehabilitation strategies used complementary in the treatment of dysphagia. The first one is the classical approach of manipulation of the biomechanical processes involved in swallowing. This includes adaptative techniques such as dietary changes and compensatory techniques, such as posture changes, various techniques of swallowing, voluntary exercises, medications and surgical procedures.

Adaptative techniques include simple measures of changing diet towards firm, thickened and possibly cold foods (2). Aliments must be homogenous, with adequate viscosity, palatability and high nutritional power.

The compensatory techniques consists in the use of specific postures and training exercises of muscles involved in the swallowing process. Specific postures beneficial for swallowing are turning the chin downward, turning the head to one side or extension of the head. These postures aimed to redirect the biomechanics of bolus flow. Training exercises will be performed initially without food ; are mobility exercises for larynx, pharynx, tongue, cheeks, lips, soft palate and vocal folds: the Lee Silverman Voice Treatment improves voice intensity and swallowing. Other maneuvers include placing the tongue between the teeth, dry swallowing, forced coughing, Valsalva maneuver, the super-glottic and super-supra-glottic swallow technique, Mendelsohn maneuver and effortful swallow maneuver (15).

These exercise programs aim to strengthen the force of the anatomical mechanisms of swallowing, working at the muscle level.

The second approach in swallowing rehabilitation address the organization and function of neural networks and is based on neuromodulatory strategies. Neuroplasticity is the mechanism by which the damaged brain regains "lost function", in response to rehabilitation (2). Neuromodulatory strategies aim to stimulate brain's capacity of reorganizing neuronal circuits in order to recover lost function (16). The techniques that modify the excitability of cortical motor representations of muscles involved in swallowing are those applied to the sensorimotor system in the periphery (thermal or tactilthermal stimulation of facial muscles, taste stimulation, swallowing related biofeedback, neuromuscular electrical stimulation and even exercises like effortful swallowing and Mendelsohn maneuver) or those applied directly to the brain (transcranial magnetic stimulation TMS).

The thermal tactile oral stimulation (TTOS) is an established method to treat patients with neurogenic dysphagia especially if caused by sensory deficits. It consists in cold stimulation of the anterior faucial pillars. It may lead to a facilitation of both the oral and the pharyngeal phase of deglutition, producing also increased bihemispheric activation with predominant activation of the left somatosensory cortical areas during the whole swallowing interval (19)

The enhancement of neural circuits of swallowing by sensory stimulation have been demonstrated by studies using functional MRI. Babaei and collab (17) tested swallowing after taste stimulation with intense flavors concomitant with presentation of olfactive and visual characters of same aliments, during fMRI scanning.Flavor increases cortical activation in the swallowing neural network: greater intensity and extension of activated cortical areas compared with dry swallowing ave been demonstrated, showing that cortical swallowing network activity can be increased by food-related sensory stimuli .

The methods that use biofeedback are of great value in rehabilitation programs: any reference for the patient to objectively 
measure their own performance and progress has proven to be useful. Many studies used biofeedback techniques to improve swallowing exercises. Swallowong movements are internal and not visible; biofeedback is used to increase swallowing biomechanic circuit. Studies use auditory feedback (hearing swallowing sounds), visual feedback (watching own laryngeal movements and normal movements), during swallowing exercises (18). Biofeedback is also combined with novel swallowing treatment maneuvers such as effortful swallowing or Mendelsohn maneuver (23). Functional MRI performed during the tests showed activation of different swallowing network areas during each of the biofeedback modalities. The use of biofeedback techniques is shown to stimulate swallowing related brain areas and can be efficient in the treatment of dysphagia.

Surface electrical stimulation of pharyngeal muscles was performed with bipolar surface electrodes placed on the skin overlying the submandibular and laryngeal regions. When electrical stimulation is applied to the skin or oral mucosa at low current levels it activates the sensory nerve endings in the surface layers providing sensory feedback to the central nervous system. (20). With increased current amplitude, the electric field may depolarize nerve endings in muscles lying beneath the skin surface and may spread to produce muscle contraction. Aspiration was significantly reduced only with low sensory threshold levels of stimulation and not during maximum levels of surface electrical stimulation, showing that the results may be related to the activation of the neural circuits involved in swallowing.

Transcranial magnetic stimulation (TMS) have also been used in dysphagia treatment. Changes in excitability of cortical projections to various swallowing muscles have been documented in response to treatments with TMS induced motor evoked potentials (MEPs). Beneficial effects of $5 \mathrm{~Hz}$ rTMS on swallowing motor cortex were observed following a repeated application of the technique over a period of weeks (21), (22).

In conclusion, swallowing rehabilitation remains a challenging problem. With actual advances in neuroimaging techniques, our knowledge of the anatomy and physiology of swallowing has increased. Better understanding of the hemispheric areas involved in swallowing control and of the sensory inputs that can modify cortical activation will provide future tools for rehabilitation treatments. The development of novel rehabilitative approaches to drive beneficial changes in cortical and subcortical activity, hence promoting improved swallowing function, remains a major imperative in dysphagia treatment.

\section{BIBLIOGRAPHY}

1. PattiF,Emmi N, Restivo DA, Liberto A, Pappalardo A, Torre LM, Reggio A. Neurogenic dysphagia: physiology, physiopathology and rehabilitative treatment. Clin Ter.2002 NovDec;153(6):403-19.

2. Bartulli FN, Luciani F, Marino S, Bramanti E, Cecchetti F, Arcuri C. The evaluation of dysphagic syndrome, in patients with previously acquired brain damae. Oral Implantol (Rome).2010 AprJun;3(2):29-34

3. Nazarko L. The clinical management of dysphagia in primary care. Br J Community Nurs. 2008 Jun;13(6):258, 260, 262-4.

4. Wieseke A, Bantz D, Siktberg L, Dillard N. Assessment and early diagnosis of dysphagia. Geriatr Nurs.2008 NovDec;29(6):376-83.

5. Matsuo K, Palmer JB. Anatomy and Physiology of Feeding and Swallowing Normal and Abnormal.Phys Med Rehabil Clin N Am. Nov 2008; 19(4): 691-707. 
6. Buchholz DW. Dysphagia associated with neurological disorders. Acta Otorhinolaryngol Belg. 1994;48(2):143-55 7. Olszewski J. Causes, diagnosis and treatment of neurogenic dysphagia as an interdisciplinary clinical problem. Otolaryngol Pol.2006;60(4):491-500

8. Paradowski L. Manometric examination in diagnosis of esophageal motility disorders.Pol Tyg Lek. 1996 Apr;51(1418):242-5. Review. Polish.

9. Ruoppolo G, Romualdi P, Formisano R, Amitrano A, Benvegnù B, Fanucci A, Metastasio F. Rehabilitation of oropharyngeal dysphagia of neurogenic etiology using radiological examination: preliminary results. Acta Otorhino to the activatiolaryngol Ital. 1992;12(Suppl 36):127

10. Hiiemae KM, Palmer JB. Food transport and bolus formation during complete feeding sequences on foods of different initial consistency. Dysphagia. 1999;14(1):31-42

11. Nishino T, Yonezawa T, Honda Y. Effects of swallowing on the pattern of continuous respiration in human adults. Am Rev Respir Dis. 1985 Dec;132(6):1219-1222

12. Palmer JB, Monahan DM, Matsuo K: Rehabilitation of Patients with Swallowing Disorders. In: Braddom R (Ed): Physical Medicine and Rehabilitation. Philadelphia: Elsevier, 2006, pp. 597-616.

13. Rosenbek JC, Robbins JA, Roecker EB, Coyle JL, Wood JL. A penetration-aspiration scale. Dysphagia. 1996;11(2): 93-98

14. Malandraki GA, Johnson S, Robbins J.Functional Magnetic Resonance Imaging of Swalowing Function: from Neurophysiology to Neuroplasticity. Head Neck.Oct 2011;33(0 1):S14-S20.

15. Nilton Felix V, Alves Correa SM, Soares RJ. A therapeutic maneuver for oropharyngeal dysphagia in patients with Parkinson's disease.Clinics.Oct 2008;63(5):661-666

16.Doeltgen SH, Huckabee ML. Swallowing neurorehabilitation: from the research laboratory to routine clinical application. Arch Phys Med Rehabil.2012
Feb;93(2):207-13.

17. Babaei A, Kern M, Antonik S, Mepani R, Ward BD, Li SJ, Hyde J, Shaker R. Enhancing effects of flavored nutritive stimuli on cortical swallowing network activity. Am J Physiol Gastrointest Liver Physiol. 2010 Aug;299(2):G422-9

18. Kawai $\mathrm{T}$, Watanabe $\mathrm{Y}$, Tonogi $\mathrm{M}$, Yamane GY, Abe S, Yamada Y, Callan A. Visual and auditory stimuli associated with swallowing: an FMRI study. Bull Tokyo Dent Coll. 2009;50(4):169-81

19. Teismann IK, Steinsträter O, Warnecke $\underline{T}$, Suntrup S, Ringelstein EB, Pantev C, Dziewas R. Tactile thermal oral stimulation increases the cortical representation of swallowing. BMC Neurosci. 2009 Jun 30;10:71

20. Ludlow CL, Humbert I, Saxon K, Poletto C, Sonies B, Crujido L. Effects of surface electrical stimulation both at rest and during swallowing in chronic pharyngeal dysphagia. Dysphagia. 2007 Jan;22(1):1-10.

21. Michou E, Mistry S, Jefferson S, Tyrrell $\underline{\mathrm{P}}$, Hamdy S. Characterizing the mechanisms of central and peripheral forms of neurostimulation in chronic dysphagic stroke patients. Brain Stimul. 2014 JanFeb;7(1):66-7

22. Park J.W., Oh J.C., Lee J.W., Yeo J.S., Ryu K.H. The effect of $5 \mathrm{~Hz}$ high-frequency rTMS over contralesional pharyngeal motor cortex in post-stroke oropharyngeal dysphagia: a randomized controlled study. Neurogastroenterol Motil. 2013;25(4) 324e250

23. Humbert IA, Suresh J. Tactile, gustatory, and visual biofeedback stimuli modulate neural substrates of deglutition. Neuroimage.Jan 16,2012;59(2):1485-1490 